


\section{La backpack como símbolo: El caso de la Facultad de Idiomas de la UABJo, México}

Erika Santiago

En este trabajo se explora el valor simbólico de la backpack

(mochila que se porta en la espalda), con base en un estudio de caso. El trabajo de campo se realizó en la Facultad de Idiomas de la Universidad Estatal de Oaxaca (UABJO), México, durante el periodo comprendido entre febrero y junio del 2013. Mediante la observación minuciosa de los usos que un grupo determinado de estudiantes dan a este objeto, se pretende desentrañar sus posibles significados más allá de la función normativa de portar las pertenencias.

Palabras clave: backpack, mochila, valor simbólico, estudiantes, función normativa 

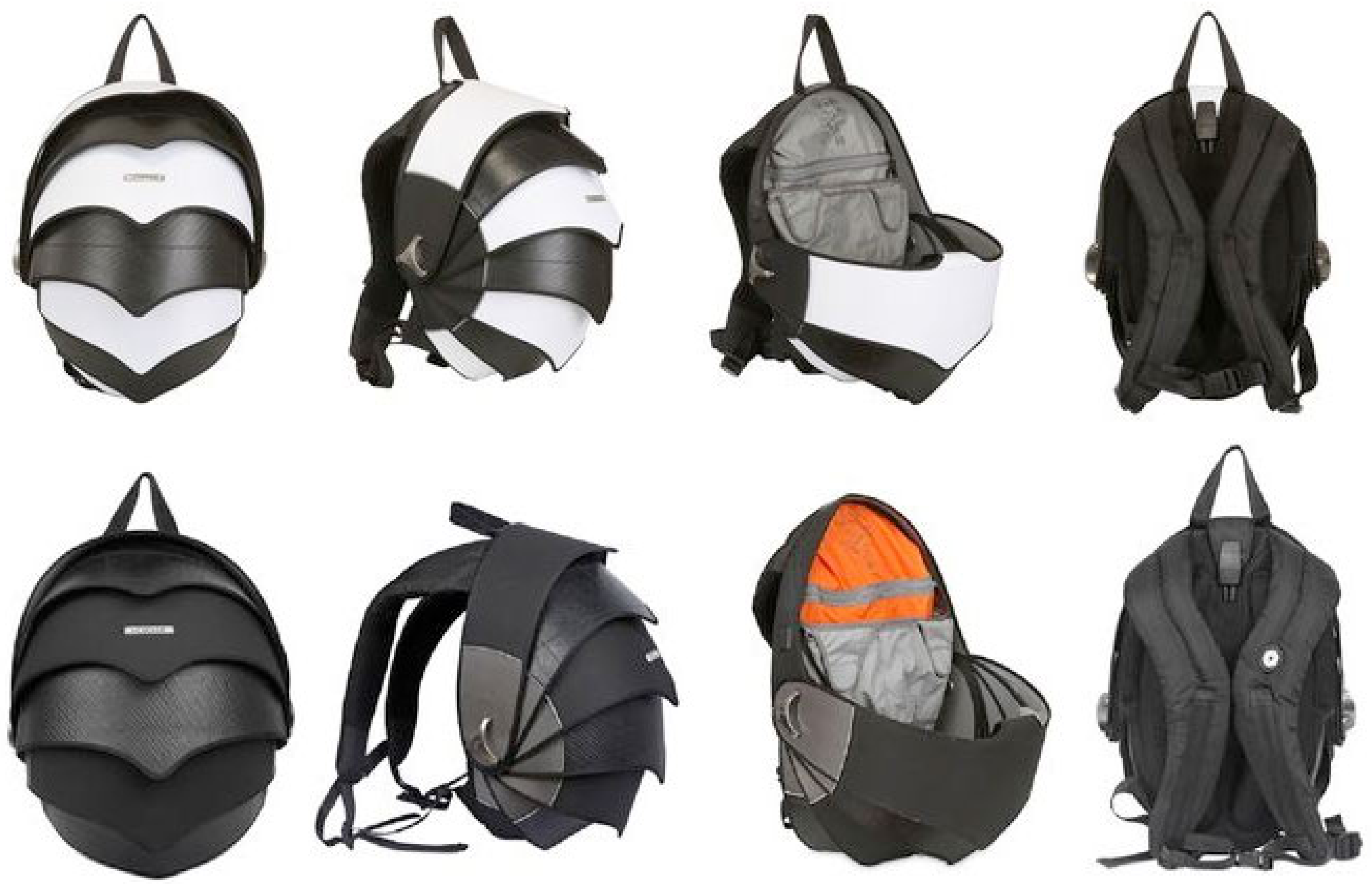

http://coolpile.com 
La backpack es un objeto de uso cotidiano que pese a contar con valor instrumental —su forma prescribe maneras específicas de utilizarlo como contenedor portátil de otros objetos- admite variaciones que se relacionan con la identidad del usuario. El estudio, que comenzó con una observación simple acerca de cómo los sujetos se relacionan con sus mochilas, se plantea como objetivo la exploración de los valores simbólicos asociados con la backpack, con miras a responder las siguientes preguntas de investigación: ¿qué significa este objeto en un contexto cultural específico? y ¿qué creencias, valores y deseos están asociados con este objeto?

La primera exploración evidenció que son más los varones que usan backpack, que los jóvenes tienden a ocuparla más que los adultos, que existen prescripciones tácitas en relación al tipo de indumentaria que se combina con la mochila y que su uso es más recurrente entre peatones que entre personas con automóvil. Todo ello constituye un indicio superficial de ciertas convenciones que se expresan en la conducta, cosa que no debe sorprendernos considerando que el hecho de transportar objetos (acto que nos remite a nuestros antepasados nómadas), así como de confeccionar y usar objetos ex profeso para ello, constituye un comportamiento culturalmente mediado. Pero, ¿qué significa este objeto?, ¿de qué nos habla?, ¿a qué creencias y valores nos refiere más allá de lo que se observa a simple vista?

La backpack o mochila fue originalmente definida como una "caja de tabla delgada, forrada de cuero, que usan los soldados para llevar el equipo, poniéndosela en la espalda, sujeta con correas y afianzada en los hombros" (DRAE, 2011). La forma en que está diseñada permite una distribución adecuada del peso, lo cual facilita el transporte de cosas en su interior.

Indudablemente, la backpack es un producto de la cultura. Es esta urdimbre de significados (Geertz, 1973, p. 20) la que motivó a los humanos primitivos para transformar una gran hoja flexible en un morral para transportar la caza del día. Por lo tanto, para desentrañar su sentido, necesitamos reflexionar en torno a dicho concepto; para el antropólogo funcionalista Bronislaw Malinowski, "la cultura es al mismo tiempo el mecanismo mínimo para la satisfacción de las necesidades de la naturaleza animal del ser humano, y también un sistema siempre en desarrollo de nuevos propósitos, nuevos valores y nuevas posibilidades creativas"(1962, p. 196). Para el autor, la cultura es un todo de carácter instrumental, esto es, media todos los aspectos del comportamiento, de modo que los seres humanos generan y reproducen la cultura, expresándola en sus técnicas, conocimientos, recreación, arte y religión (p. 199). La cultura se expresa en nuestro sentido común como un "sistema ins- trumental total de actividades organizadas" (ibid), actividades que abarcan la creación y el diseño. En este orden de ideas, la backpack es un producto de la cultura que se relaciona con la propiedad privada y su uso implica una serie de reglas y expresa los valores vigentes en el contexto en el cual se utiliza.

Para desentrañar los valores asociados con el objeto de interés, el estudio abreva de las aportaciones de Roland Barthes (1978) al respecto de la naturaleza del texto, que a su vez se vincula estrechamente con las aportaciones de Louis Hjelmslev (1961) en lo relativo a los niveles de expresión y de contenido. Para Barthes, es posible descomponer el texto en niveles denotativo (descriptivo) y connotativo (de contenido profundo) y esta descomposición, a la cual se suma la función del texto escrito, permite que el analista descubra el mecanismo de reloj que hace posible el complejo fenómeno de la significación.

En el caso que nos ocupa, partimos del supuesto de que cualquier objeto puede ser interpretado como texto, toda vez que la observación se descomponga y sistematice con base en los niveles referidos. A este enfoque debemos sumar la propuesta de González y Yuste (1998, p. 180), quienes proponen ocho funciones de los objetos que poseemos. La primera es la función utilitaria que aplica a todos los objetos. De la segunda en adelante se involucran valores simbólicos como estructuras sociales y dominio social, motivación de afecto, dominio y control de uno mismo y del mundo, identificación personal, definición del "yo" y elementos del pasado y del futuro (1998). Más adelante se ilustrarán estos niveles.

\section{Discusión}

Para abordar el caso que nos ocupa, se diseñó una entrevista semiestructurada que constó de dos reactivos abiertos y siete cerrados, tendientes a identificar los usos más comunes, así como los significados profundos que el usuario deposita en su backpack. La entrevista se aplicó en el mes de abril a estudiantes que fueron contactados en las instalaciones de la Facultad de Idiomas de la UABJO en horas de entrada y salida de clases y en lugares donde se reúnen durante su tiempo libre. Para diseñar la entrevista se consideraron las siguientes categorías de análisis: edad, género, formas usos del objeto, frecuencia de uso, practicidad, preferencias de diseño. Las dos preguntas abiertas estuvieron orientadas a conocer la relación de los usuarios con su mochila más allá del aspecto funcional. El propósito fue explorar el apego emocional relacionado con el valor simbólico, en otras palabras, los lazos emotivos que existen entre la backpack y su usuario.

Con base en una observación preliminar, se decidió que los informantes fueran estudiantes universitarios de la Facultad de Idiomas de la UABJO. Para la recolección de datos, observé la diná- 


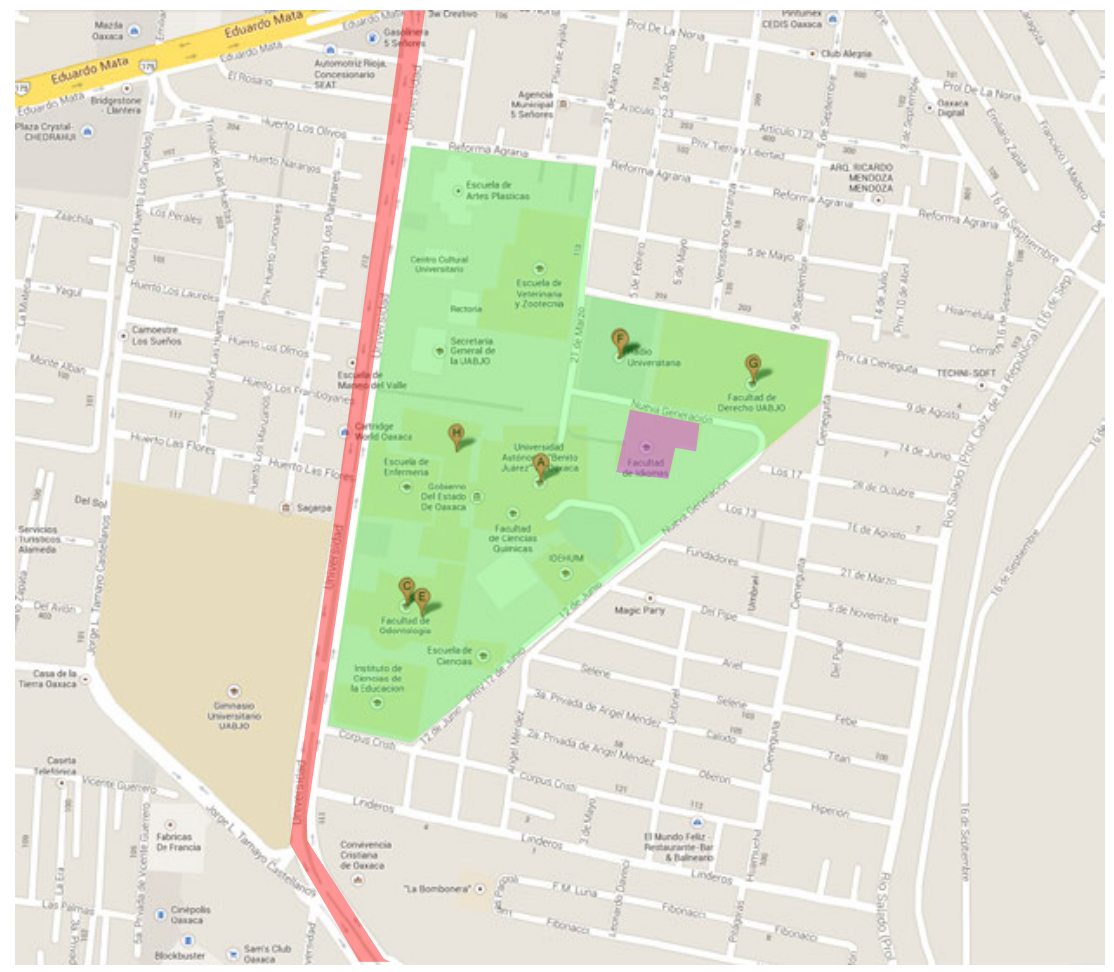

Universidad Estatal de Oaxaca (UABJO)

Facultad de Idiomas de la UABJO

- Ruta de camiones urbanos

Figura 1
La Ciudad Universitaria no cuenta con sitio de taxis, el más cercano se sitúa a 3 cuadras en una plaza comercial. Existen algunas rutas de camiones que pasan por avenidas cercanas a la universidad. Debido al escaso transporte urbano, muchos estudiantes optan por caminar. En la figura 1 se ubica la universidad y las rutas de autobuses más cercanas.(figura 1)

Como se mencionó antes, la pregunta principal que se plantea en este estudio es: ¿Qué significado, en términos de creencias, valores y deseos, dan a la backpack los estudiantes de la Facultad de Idiomas de la UABJO? Veamos la manera en que los participantes de este estudio respondieron a esta interrogante en la figura 2. La mayor parte de los entrevistados consideraron que la backpack es muy importante, si bien no indispensable, al igual que los objetos que ésta contiene; su uso es más recurrente en la escuela y se asocia con la practicidad y la seguridad. Aunque aparentemente no se trata de un objeto indispensable cuya pérdida se lamentaría, los usuarios tienden a considerar que su backpack tiene un carácter único, resultado de la combinación de su contenido y de las decisiones que el poseedor toma para apropiarse del objeto, lo mismo en su aspecto que en su forma de portar la mochila. (figura 2)

Las respuestas más frecuentes se sistematizaron en coherencia con la propuesta de González y Yuste (1998) y pueden observarse en la figura 3. Una constante durante las entrevistas fueron las anécdotas referidas por los usuarios para resaltar el carácter único de su backpack.

Cuando se solicitó a los usuarios explicar el motivo de la asociación entre el uso de la mochila y la seguridad (menos obvio que el vínculo entre ésta y la practicidad de llevar los objetos de interés en la espalda), los usuarios hicieron referencia a compartimentos ocultos que suelen usar para esconder sus objetos más valiosos. Esto es, los atributos del objeto nos hablan del contexto sociocultural y de los valores de la época, entre los cuales destaca la seguridad -o visto desde la perspectiva del contexto, la prevalencia de la inseguridad en el entorno y/o la percepción del entorno como inseguro por parte del usuario-.

Uno de los entrevistados comentó al respecto de su backpack, mientras nos mostraba su contenido:

Traigo mochila porque hago varias cosas (...) tengo que tener todo a la mano (...) Está rota, pero no la cambio. Ya aprendí a moverme con la mochila (...) En estas bolsas traigo siempre estos [pañuelos desechables], para mi jardín de niños, nunca sabes. Traigo plumón para calificarles y un gel, la memoria [USB] y (...) papelitos. En la siguiente bolsa guardo siempre mi iPod (...) a la mano (...) como es de malla me gusta porque cuando abro 


\section{Tabla de resultados}

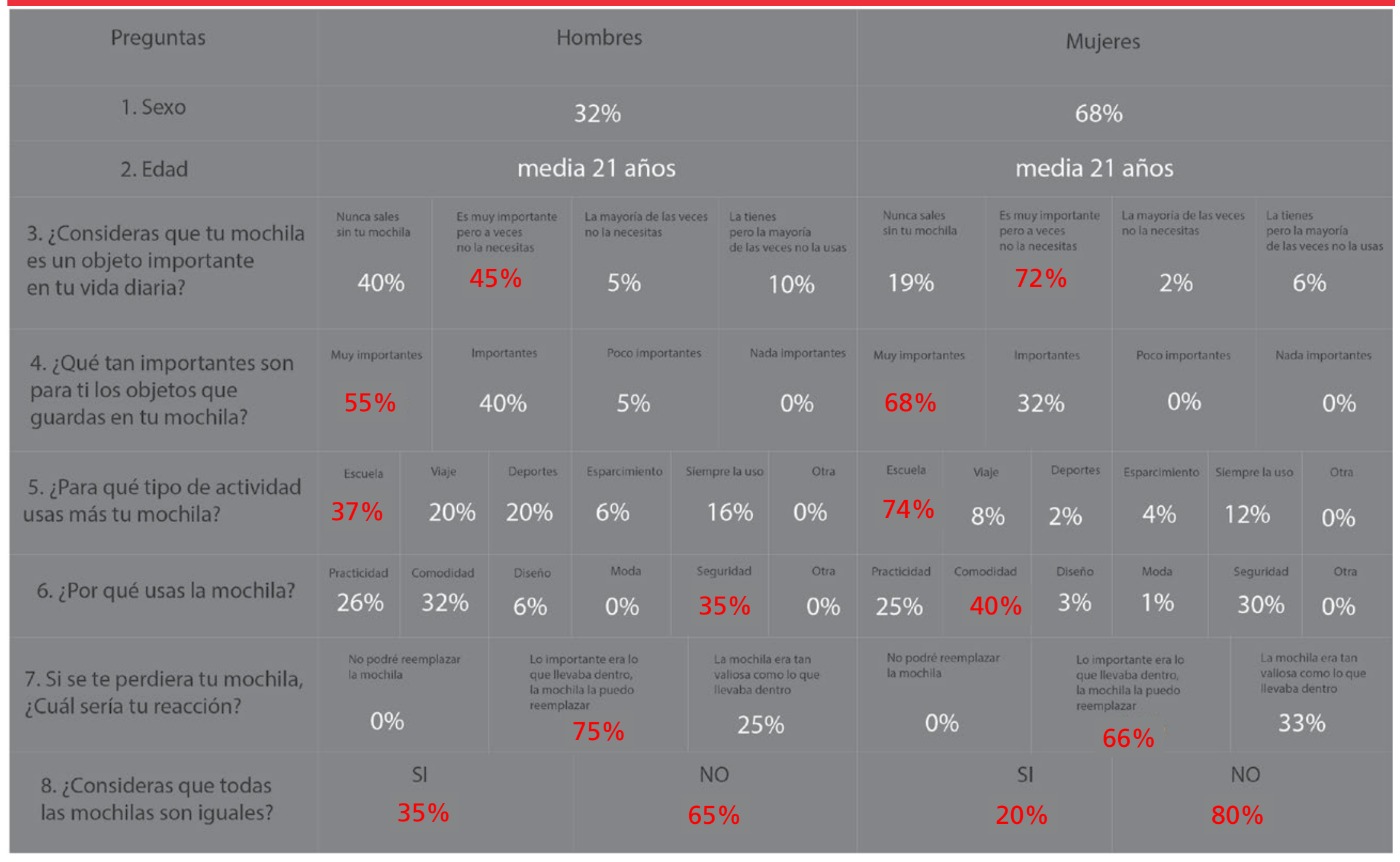

Figura 2

la mochila puedo ver si está o no está (...) quiero verlo que está ahí. Me gustan las mochilas que tienen muchas bolsitas (...) Esta es la bolsa principal pero todavía tiene otro espacio aquí... de repente cuando quiero asegurar algo (...) lo meto directamente aquí porque sé que es fácil sacarlo (...) cuando lo tengo con las otras cosas sé que se puede atorar (...) No me había puesto a pensar pero creo que la mochila siempre ha sido parte de mí (...) Cuando la traje todo mundo me dijo: "Está muy padre tu mochila". Creo que inconscientemente ya es parte de mi personalidad. De hecho también la ocupo como arma, como vivo en la central [de abastos], cuando siento que alguien viene detrás de mí, bajo discretamente la mano hasta acá [agarra la parte inferior del asa derecha de la mochila] y entonces sé que la puedo usar así [se la quita de un solo movimiento y hace como que le pega a alguien con la mochila] (...). La cargo de diferentes formas pero en la central me la pongo muy justa, yo creo que para sentirla más segura. Creo que estoy listo para vivir en el D.F. [risas]. (Federico, 29/abril/ 2013)

La descripción del entrevistado da cuenta de los niveles tanto denotativo como connotativo, bordando en el valor que tiene su mochila en los diferentes contextos en los que participa. Este ejemplo nos permite derivar en los dos significados de la mochila identificados en el estudio.

\section{La backpack como extensión de hogar}

Uno de los aspectos que saltan a la vista en las explicaciones de los estudiantes, es la manera en que la backpack, en su naturaleza de contenedor de objetos, forma parte esencial de la vida cotidiana de los usuarios. En un sentido se puede afirmar que la backpack es una extensión de la casa, una pequeña casa móvil que incluye desde artículos de vestir hasta comida: "casi siempre traigo mi comida", "me puedo quitar el suéter y lo guardo cuando hace calor", "llevo libros, mi iPod, el suéter que a veces no sabes si lo vas a ocupar o no", "guardo el líquido para limpiar mis lentes, papel de baño", incluso "la ocupo como almohada", "traigo mi agua, mi diccionario, que es como mi Biblia, un buen libro para leer en el camión, mi cuaderno para las clases, mi material que uso con los niños, lapicera, cosas que me regalan los niños y los conservo, mi lunch, papeles en los que anoto, que son importantes. Por ejemplo, traigo dibujos de mi nena de 4 años y siempre los 


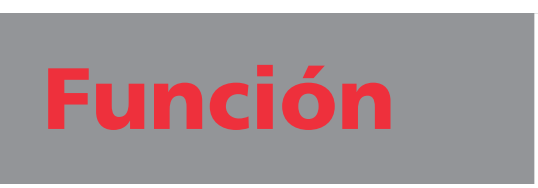

Utilitaria

\section{Reflejo de estructuras} sociales dominantes

\section{Fuente de motivación de afecto}

Dominio y control

Identificación personal

Definición del "yo"

\section{Objetos como elementos del pasado}

\section{Preguntas que aluden a la función}

"Unas brindan más comodidad que otras",

"El material aguanta más que otras"

Estos resultados son obvios, pues se podría afirmar que cualquier backpack

connota elementosde portabilidad, individualidad y practicidad.

"Es más segura que las otras"

Los elementos nombrados (diseño, marca, modelo), están relacionados con el poder de adquisición y, por consecuencia, el estatus social de la persona que porta la mochila.

"Es parte de mi vida", "me acompaña en todo momento"

Algunos participantes también expresaron que su mochila es especial porque les gusta su apariencia.

"Es más seguro que las otras". En diferentes momentos de la entevista, José me ilustró acerca de la mochilale ha ayudado a dominar los diferentes roles que le ha tocado vivir en su vida de estudiante, maestro, padre de familia y deportista.

Es interesante notar como dice José dice que "mi mochila" (y no él) "tiene un orden", adjudicándole a la mochila ese control que tiene sobre el contenido de la misma.

"Por ser mia ya es especial", "el tamaño que se ajusta a mis necesidades, "tiene los compratimentos que necesito para guardar todas mis cosas", "satisface mis necesidades"

"Porque me describe como persona" o implícita: "me la regaló una persona especial para mi": "Yo la elegí", "Me recuerda momentos únicos y experiencias".

"Tuve una experiencia religiosa de dos años y la llevé todos los días a la calle", "la perdí en una borrachera", " estaba conmigo viajando", "me ha acompañado desde mi adolescencia". Otros las relacionan con personas, "ha estado conmigo conociendo mujeres diferentes", "una vez con mis amigos pensé que la había perdido", "le cuelgo cosas que me regalan los niños".

Finalmente las anécdotas que tiene que ver con la funcionabilidad "ha tenido descomposturas, la he cosido y parchado", "traía mucho dinero, puro cambio para el camión por eso se rompió". 
llevo, hasta chicles para el aliento (...) he metido hasta biberones y pañales en mi mochila, lo que fuera necesario cargar, practico futbol y eso como playeras y agua también lo llevo".

\section{La backpack como extensión de sujeto}

Los entrevistados coincidieron en el valor utilitario de la backpack como su primer vínculo con el objeto sin embargo, el proceso de seleccionar la que más les llamaba la atención transformó el vínculo, añadiéndole componentes emocionales: "Cuando la fui a escoger, tardé como quince días para comprarla, primero me fijé bien del color y que le cupiera mucho, vi como tres cuatro, como que no me convencí y volví a verla otro día", "la vi y me gustó. Creo que la compré en Coppel. Me gustó en primer lugar el color negro".

La relación que inicia con una mirada en el fondo de la tienda, un encuentro inesperado en el escaparate o el debate interno por dos semanas seguidas, se transforma en una forma de hablar del objeto como si tuviera cualidades humanas ya sea siendo parte de uno mismo, definiendo al "yo"(González y Yuste, 1998): "Creo que la mochila siempre ha sido parte de mí", "inconscientemente ya es parte de mi personalidad y de cierta manera refleja mi personalidad soy muy abierto", "si mi mochila fuera una persona sería fiel, como una amiga", "mi mochila (...) es una persona que le dices apóyame y ahí está".

De diferentes maneras, los participantes en esta investigación nos hicieron partícipes de dos formas en que, entre muchas otras, conciben al objeto estudiado: como una extensión de la casa y como un ente que los acompaña o incluso que constituye una extensión del sujeto. La importancia de la backpack no es la de un objeto más que se trae consigo, sino como un metaobjeto (un objeto que contiene otros objetos) que porta, acompaña y comparte atributos con quien lo posee:

Al mismo tiempo mi backpack es mi compañera. La relación que he establecido con ella no la considero ni superficial ni pasajera. Ella no sólo me brinda comodidad y seguridad, sino también compañía. Por lo tanto, lo menos que puedo hacer por ella es considerarla y tratarla bien, por eso nunca la pongo en el piso y le doy mi lugar ("tiene su silla, yo no me siento pero se sienta ella"). Además es un testigo de mis experiencias en la vida. Ha estado siempre presente en lo que me pasa, experimentando, como yo, disputas y alegrías. Además, le doy el lugar y el respeto que se merece, no importa que los demás la vean rota o descompuesta. Tampoco me interesa la opinión negativa de los demás, pues algunos piensan que ya la tengo que lavar, pero a mí me interesa más que no se le vaya a gastar el color.

\section{Conclusiones}

A pesar de las limitaciones del estudio (el tiempo de observación, la interacción con los participantes), la información recabada me permitió ver al objeto estudiado: la backpack, de otra manera. La exploración arrojó nuevas interrogantes, por ejemplo en lo que refiere a la estrecha relación entre su uso, la actividad realizada por el propietario (en este caso, estudiantes) y el acceso a un medio de transporte; ésta última variable es, sin duda, uno de los temas de interés que pueden explorarse en estudios subsiguientes.

El otro resultado relevante refiere, como ya he señalado, al valor de la mochila más allá de su utilidad como objeto que sirve para transportar y mantener la cercanía de otros objetos. Sin duda, al interpretarse como texto, la backpack revela su carácter como extensión del hogar y como acompañante de quien la porta, valores que no se relacionan de forma obvia con su función primordial.
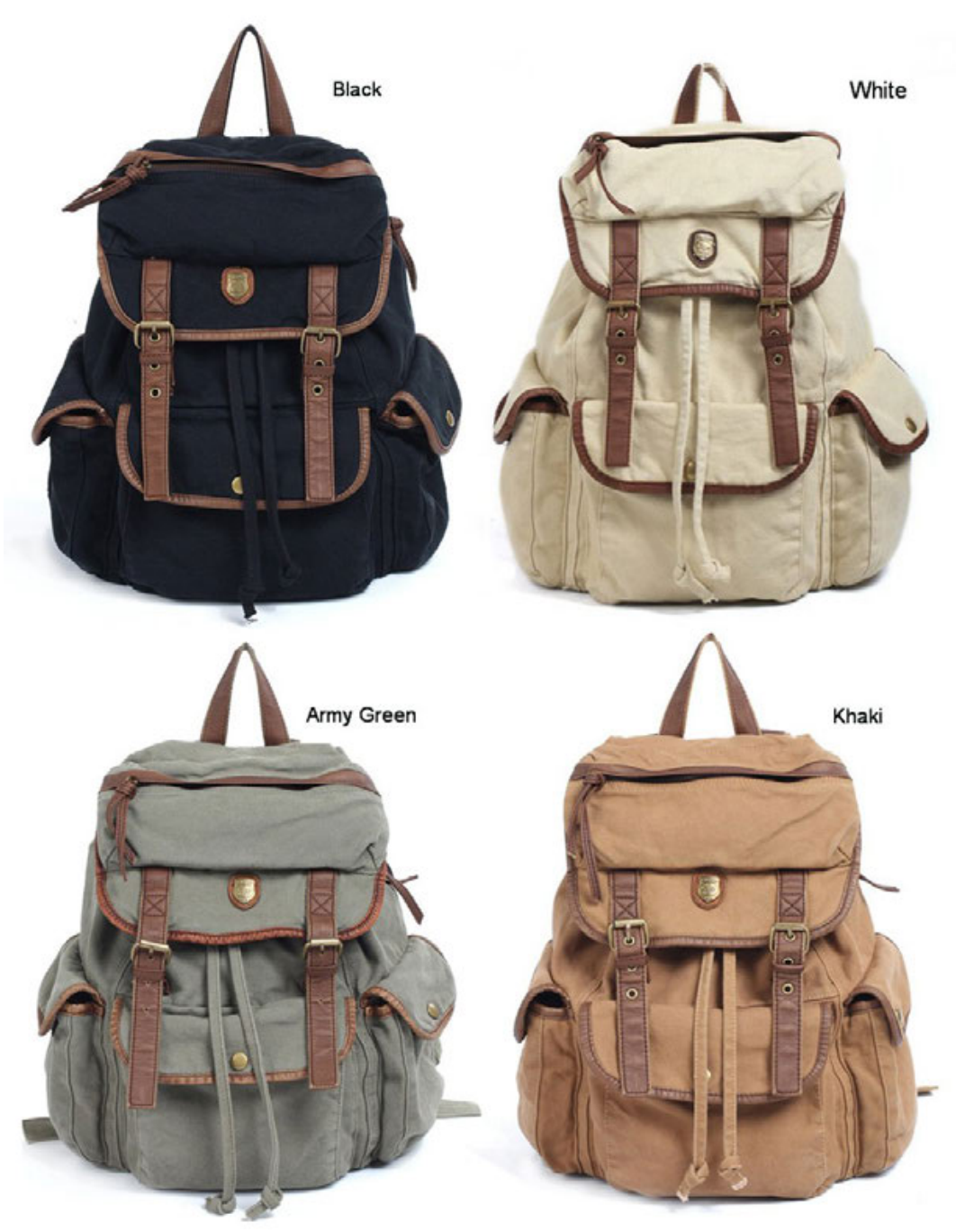

http://es.shopmadeinchina.com/ 


\section{Referencias}

Acosta, L. (2010). La estética de la frivolidad. Moda y representaciones contemporáneas. Razón y palabra, 72.

Agullo, E., y Rodríguez J. (1999). Estilo de vida, cultura, ocio y tiempo libre de los estudiantes universitarios. Psicothema, 11, 2, 247-259.

Appadurai, A. (1986). The social life of things. Commodities in cultural perspective. Cambrige, Cambrige University Press.

Barthes, R. (1978). Image, Music, Text. New York, Hill and Wang.

Camacho, A. (2006). Socioepistemología y Prácticas Sociales. Educación Matemática, 18, 001, 133-160

Castro, G. (2005). Los jóvenes y la vida cotidiana, elementos y significados de su construcción. Espacio abierto, 14,001, 7-23.

Crown, D. (2003). Visible Signs. Switzerland, Ava Publishing.

Csikszenymihalyi \& M.Rochberg-Halton, E. (1981). The meaning of things, domestic simbols and the self. Cambrige, Cambrige University Press.

De Saussure F. (1974). Course in General Linguistics. Fontana.

Oxford University Press, Diccionario Oxford, (2013), recuperado el 8 de marzo del 2013, http://oxforddictionaries.com

Fabric \& handle (2011), Backpack history, How and when dis they came into being?, recuperado el 9 de marzo del 2013 de www.fabric-and-handle.com/articles/backpack-history---/howand-when-they-came-into-being/

Gaytán, A. (2011).Calle, cuerpo y género. La identidad como proceso en la ciudad de México. Acta Sociológica, 55, 37-54.

Geertz, C. (2005). La interpretación de las culturas.

Barcelona, España: Gedisa.

González F. y Villegas M. (2011). La investigación cualitativa de la vida cotidiana. Medio para la construcción de un conocimiento sobre lo social a partir de la individual. Psicoperspectivas, 10, 2, 35- 59.
González I. y Yuste N. (1998). Los objetos personales favoritos en la adultez y senectud, una aproximación empírica. Anales de psicología, 14, 2, 177-192.

Hjelmslev, L. (1961). Prolegomena to a Theory of Language: Univ. of Wisconsin.

Illinois State Board of Education, (2006), Carrying Backpacks: Physical Effects, recuperado el 3 de abril del 2013 de http:// www.isbe.state.il.us/pdf/school_health/Carrying_Backpacks_ Physical_Effects.pdf

Malinowski, B. (1962). Sex, Cultura and Mith. Brace and World, Nueva York.

Mauss, M. (1971). Sociología y Antroplogía. Madrid, Tecnos.

Mikel (2012). Historia de la mochila. recuperado el 3 de abril del 2013 de http://armysoft.es/blog/index.php/historia-de-lamochila/

Morales A. (2010), Sobre leyendas, recuperado el 29 de agosto del 2013 de sobreleyendas.com/2008/06/12/la-sonrisa-detepoztecat//

Morales D. y Ramírez E. (2011). Análisis representacional de los valores de las personas que usan ropa de marca. Entornos, 24, 147-159.

Norman, D. (2005). Diseño emocional. Por qué nos gustan o no los objetos cotidianos. Barcelona, España: Paidós.

Perret R. (2010). Todos con la camiseta bien puesta. Mundo ejecutivo, 110-111.

Real academia española,(2011), recuperado el 26 de abril de abril de www. rae.com.es

Tamayo, E. (2010). La función de los objetos. Grafías, 12, 31-33.

Vigotsgy, L. (1977). Pensamiento y Lenguaje. Buenos Aires, La Pléyade

Zambrini, L. (2010). Prácticas del vestir y cambio social. La moda como discurso. Revista online: Questions, 24. 